From the Division of Hematology and Oncology, Department of Medicine, The University of North Carolina at Chapel Hill, Chapel Hill, NC; and the Aab Cardiovascular Research Institute and Department of Medicine, University of Rochester, Rochester, NY.

Submitted February 17, 2009; accepted June 22, 2009; published online ahead of print at www.jco.org on September 8, 2009

Supported by the National Institutes of Health and the University of North Carolina Cancer Research Fund.

Authors' disclosures of potential conflicts of interest and author contributions are found at the end of this article.

Corresponding author: Nigel Mackman, PhD, University of North Carolina at Chapel Hill, 917 Mary Ellen Jones Bldg, CB7035, Chapel Hill, NC 27599; e-mail: nmackman@med.unc.edu.

(C) 2009 by American Society of Clinical Oncology

0732-183X/09/2729-4834/\$20.00

DOI: $10.1200 / J C O .2009 .22 .6324$

\title{
Role of Tissue Factor in Cancer
}

Raj S. Kasthuri, Mark B. Taubman, and Nigel Mackman

$$
\begin{array}{llllllll}
\text { A } & \text { B } & \text { S } & \text { T } & \text { R } & \text { A } & \text { C } & \text { T }
\end{array}
$$

Tissue factor (TF) is a transmembrane glycoprotein that localizes the coagulation serine protease factor VIINIIa (FVIINIIa) to the cell surface. The primary function of TF is to activate the clotting cascade. The TF:FVIla complex also activates cells by cleavage of a G-protein coupled receptor called protease-activated receptor 2 (PAR2). TF is expressed by tumor cells and contributes to a variety of pathologic processes, such as thrombosis, metastasis, tumor growth, and tumor angiogenesis. For instance, tumor cells release TF-positive procoagulant microparticles into the circulation and these may trigger venous thromboembolism in patients with cancer. TF on circulating tumor cells also leads to the coating of the cells with fibrin that traps them within the microvasculature and facilitates hematogenous metastasis. In addition, TF:FVIla-dependent activation of PAR2 on tumor cells increases tumor growth via an undefined mechanism. One possibility is that PAR2-dependent signaling increases the expression of proangiogenic proteins. Other studies have reported that endothelial cells in the tumor vasculature express TF and this may enhance angiogenesis. These results suggest that inhibition of TF should reduce several pathologic pathways that increase tumor growth and metastasis. This would represent a novel approach to anticancer therapy. Initial studies using inhibitors of the TF:FVIla complex in mouse tumor models have produced encouraging results. Nevertheless, additional studies are needed to determine if this strategy can be successfully translated to the treatment of cancer patients.

\section{J Clin Oncol 27:4834-4838. (c) 2009 by American Society of Clinical Oncology}

\section{INTRODUCTION}

Tissue factor (TF) is a transmembrane glycoprotein that is essential for hemostasis. It binds the coagulation serine protease factor VII/VIIa (FVII/VIIa) to form a bimolecular complex that functions as the primary initiator of coagulation in vivo. ${ }^{1}$ The TF/ FVIIa complex activates both FX and FIX and leads to the generation of thrombin and fibrin. Thrombin also activates platelets by cleavage of proteaseactivated receptors (PARs). ${ }^{2} \mathrm{TF}$ is constitutively expressed at high levels in adventitial fibroblasts of the vessel wall and this facilitates the rapid activation of the coagulation cascade after injury. ${ }^{3,4}$ TF can also be induced in the smooth muscle cells and endothelial cells of the vessel wall under various pathologic conditions, ${ }^{5,6}$ resulting in a prothrombotic state. TF is also expressed in a tissue-specific manner with high levels in a variety of tissues, such as the brain, heart, kidney, and placenta. ${ }^{7}$ This pattern suggests that TF provides additional hemostatic protection to these vital organs. In support of this notion, both the inhibition of TF in mice with normal levels of TF and mice with reduced levels of TF exhibit bleeding in certain tissues, including the heart and brain. ${ }^{7}$

Very low levels of TF are present in the blood of healthy individuals. This so-called circulating TF is mostly present in the form of microparticles (MPs), which are submicron membrane vesicles derived from apoptotic and/or activated cells. ${ }^{8}$ The role of circulating TF in healthy individuals has not been defined. ${ }^{9,10}$ Blood also contains soluble forms of TF, including alternatively spliced $\mathrm{TF} .{ }^{10}$ However, the absence of the transmembrane domain greatly reduces the procoagulant activity of this alternatively spliced $\mathrm{TF}^{11}$

Many studies have shown that levels of circulating TF in the form of MPs are increased in various diseases, including cardiovascular disease, sepsis, diabetes, and cancer. ${ }^{8}$ This MP TF likely contributes to the increased incidence of thrombosis observed in these diseases. One study showed that TF-positive MPs isolated from the pericardial blood of cardiac surgery patients increased venous thrombosis in the rat model. ${ }^{12}$

In endotoxemia and sepsis, TF expression by cells within the vasculature leads to disseminated intravascular coagulation. Monocytes appear to be the major source of intravascular TF in many diseases, such as sepsis. For instance, bacterial lipopolysaccharide induces monocyte TF expression and the release of TF-positive MPs in vitro and in vivo. ${ }^{13,14}$ We found that decreasing TF expression in hematopoietic cells reduced the activation of coagulation in 
a mouse model of endotoxemia. ${ }^{15}$ Other cell types, such as endothelial cells, can also express TF in vitro. ${ }^{16}$ In addition, TF staining of endothelial cells has been observed in the splenic microvasculature and at branch points in the aorta of septic baboons. ${ }^{17,18}$ However, it is unclear if this staining is due to TF expression by the endothelial cells themselves or deposition of leukocyte-derived, TF-positive MPs. We have found that selective deletion of the TF gene in endothelial cells does not reduce the activation of coagulation in endotoxemic mice (Pawlinski and Mackman, unpublished data).

$\mathrm{TF}$ is expressed by all types of cancers. Importantly, TFdependent activation of coagulation has been implicated in cancerassociated thrombosis and metastasis (Fig 1). In addition to its procoagulant activity, TF has cell signaling properties. Formation of the TF:FVIIa complex on the surface of tumor cells leads to cleavage and activation of the G-protein-coupled receptor PAR2. The TF: FVIIa-PAR2 signaling pathway appears to promote tumor growth and tumor angiogenesis (Fig 1). This review will discuss how TF expression by tumor cells may contribute to thrombosis, metastasis, tumor growth, and tumor angiogenesis.

\section{TF AND THROMBOSIS}

The incidence of thrombosis is significantly increased in patients with cancer compared with the general population. Cancer is associated with a four-fold increase in the risk for venous thromboembolism (VTE) and chemotherapy further increases this risk to between six and seven-fold compared with that in the general population. ${ }^{19}$ Overall, the occurrence of VTE in patients with cancer is associated with increased morbidity and mortality. ${ }^{20}$ The epidemiology of cancerassociated thrombosis is discussed in detail elsewhere in this issue.

Many recent reports have suggested a role for TF in the development of cancer-associated thrombosis. TF expression has been reported in a number of cancers, such as glioma, pancreatic cancer,

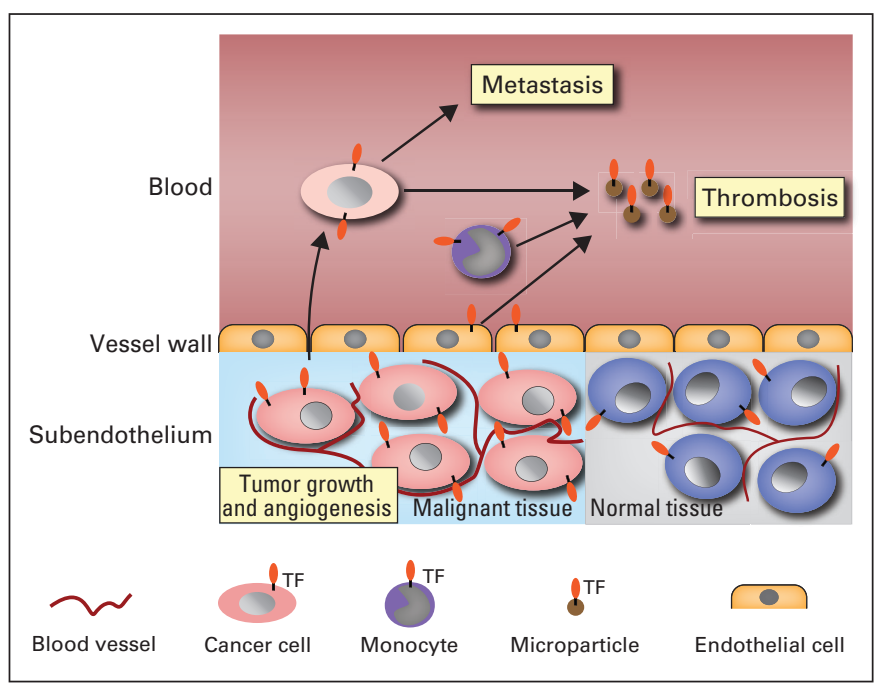

Fig 1. Tissue factor (TF) contributes to tumor growth, angiogenesis, metastasis, and thrombosis in patients with cancer. TF expression by tumor cells may increase the growth of tumors by increasing cell survival and/or increasing angiogenesis. TF expression by tumor cells in the blood enhances metastasis by activating coagulation and platelets. Release of TF-positive microparticles by tumor cells and host cells into the blood may trigger venous thromboembolism. non-small-cell lung cancer, colorectal cancer, renal cell cancer, ovarian cancer, prostate cancer, hepatocellular cancer, and breast cancer. ${ }^{21}$ The level of TF expression varies among different types of cancers and, in general, increases with advanced cancer stage. ${ }^{22}$ Interestingly, the incidence of thrombosis in patients with tumors expressing higher levels of TF, such as brain and pancreatic cancer, is greater than in those with tumors expressing lower levels of TF, such as breast cancer. ${ }^{23}$ For instance, increased TF expression in biopsies of patients with pancreatic cancer was shown to correlate with a greater incidence of VTE. $^{24}$ Similarly, increased TF expression in resected ovarian cancers was found to be an independent predictor of risk for VTE. ${ }^{25}$ There are also reports of increased TF expression in resected squamous cell carcinoma ${ }^{26}$ and increased levels of circulating TF in patients with lung cancer, ${ }^{27,28}$ although no data was provided on the incidence of VTE in these studies.

There is support for the notion that increased levels of circulating TF contributes to the prothrombotic state in patients with cancer. Tumor cells themselves are likely to be an important source of the elevated levels of circulating TF. An early study by Dvorak et $\mathrm{al}^{29}$ showed that cancer cells shed procoagulant plasma membranes (commonly referred to as MPs). The authors proposed that these MPs may account for the activation of the coagulation system associated with malignancy. Many other studies have now shown that a variety of tumor cells release TF-positive MPs. Tumor-derived TF-positive MPs may gain access to the bloodstream via leaky vessels within the tumor vasculature (Fig 1). The presence of a tumor may also elicit a host response that leads to induction of TF expression in monocytes and possibly endothelial cells, both of which are known to shed TFcontaining MPs. Currently, the relative contribution of tumor cells and host cells to this pool of circulating TF-positive MPs is unknown.

Treatment of patients with chemotherapeutic agents and/or radiation may promote TF expression and/or the release of TFpositive MPs, resulting in an increase in the levels of circulating TF. An early study reported that treatment of endothelial cells with chemotherapeutic and antiangiogenic agents increased TF activity without increasing TF expression. ${ }^{30}$ More recently, it was shown that doxorubicin and epirubicin increased TF activity in endothelial cells by inducing the exposure of the anionic phospholipid phosphatidylserine. ${ }^{31}$ However, another study reported that cisplatin induced the release of procoagulant MPs from endothelial cells that lacked TF. ${ }^{32}$ These differences appear to be due to the fact that endothelial cells can sometimes express low levels of TF depending on the culture conditions.

Mouse tumor models have been used to measure the levels of circulating TF. We found that mice containing human colorectal tumors had detectable levels of human TF antigen in the circulation. ${ }^{33}$ We noted an association between tumor size and human TF antigen levels in the plasma with higher levels noted in mice with larger tumors. In addition, tumors expressing higher levels of TF released more TF into the circulation than tumors expressing lower levels of TF. Another study with mice containing human pancreatic tumors found a positive correlation between the levels of plasma tumorderived human TF activity and thrombin-antithrombin complex, a marker of activation of coagulation. ${ }^{34}$ Taken together, these results suggest that tumors are a significant source of circulating TF that likely promotes a hypercoagulable state. Indeed, we found that low TF mice containing Lewis lung carcinomas were protected against 
cyclophosphamide-induced toxicity, ${ }^{35}$ suggesting that TF contributed to the death of these mice.

Recent studies have focused on circulating TF-positive MPs and their role in cancer-associated thrombosis in humans. One report found an association between increased levels of circulating TFpositive MPs and the incidence of VTE in patients with advanced pancreatic and breast cancer. Higher levels of circulating TF-positive MPs were also associated with decreased overall survival. ${ }^{36}$ In another study, the number of TF-positive MPs was two-fold higher in patients with advanced colorectal cancer compared with healthy controls. ${ }^{37}$ Moreover, levels of TF-positive MPs correlated with levels of D-dimer, which is derived from degraded fibrin and elevated in patients with VTE. We evaluated the association between circulating TF-positive MPs and development of VTE prospectively in 11 patients with pancreatic cancer. We found that the two patients that had the highest levels of TF antigen and MP TF activity in serial plasma samples subsequently developed VTE. ${ }^{38}$

In summary, there are emerging data that an increase in circulating TF, primarily in the form of TF-positive MPs, may be associated with an increased risk for thrombosis in patients with cancer. However, it has yet to be established whether or not levels of circulating TF can be used as biomarkers of thrombotic risk. Prospective studies are being performed to determine if levels of MP TF are predictive of VTE in patients with cancer. In addition, the use of clinical probability models to estimate the risk of VTE in cancer that incorporate biomarkers, such as MP TF, are currently in progress.

\section{TF AND HEMATOGENOUS METASTASIS}

An important mechanism of tumor metastasis is via the intravascular space-so-called hematogenous metastasis. Although such hematogenous metastasis can occur directly in the form of tumor emboli, it is likely that components of the host clotting system contribute to this process.

Several studies have shown that tumor cell TF plays an important role in hematogenous metastasis. In a mouse model of pulmonary metastasis, inhibition of TF with a variety of agents, such as anti-TF antibodies and tissue factor pathway inhibitor, reduced tumor metastasis. $^{39-41}$ Tumor TF may enhance metastasis by inducing the coating of the tumor cells with fibrin that would trap the cells in the microvasculature, thereby aiding metastasis. Thrombin also activates platelets, leading to the formation of platelet-fibrin thrombi in the microvasculature. In support of this mechanism, tumor metastasis is decreased in various mice, including those deficient in fibrinogen, and in those deficient in PAR4, which are resistant to activation of platelets by thrombin. ${ }^{42,43}$ Clinical case series have also reported a correlation between TF expression and tumor metastasis in patients with colorectal and lung cancer. ${ }^{44}$ Formation of a clot around the tumor cells in the circulation also prevents killing of the tumor cells by natural-killer cells. ${ }^{45,46}$ Thrombus formation in the microvasculature may also activate the endothelium and induce the expression of adhesion molecules that facilitate tumor cell migration into the extravascular space.

In summary, TF expression by tumor cells appears to enhance tumor metastasis, probably via activation of both the coagulation cascade and platelets. From a clinical standpoint, these observations provide a rationale for the use of antithrombotic agents as adjuvants in the treatment of patients with cancer. Clinical trials are needed to examine this prospectively in the clinical setting.

\section{TF AND TUMOR ANGIOGENESIS}

TF expression by tumor cells has been implicated in enhanced tumor growth and angiogenesis. TF has been reported to be expressed on endothelial cells within the tumor vasculature, ${ }^{47}$ although this observation has not been universal. ${ }^{48}$ One would think that increased TF expression on endothelial cells would induce thrombosis and tumor death, rather than promote tumor growth. However, TF expression by endothelial cells has been proposed to enhance angiogenesis. ${ }^{49}$ One study showed that increased TF expression in tumor cells was associated with increased expression of the proangiogenic protein vascular endothelial growth factor (VEGF) and increased tumor size in mice. ${ }^{50}$ In human tumors, high levels of TF expression are associated with increased VEGF expression and with increased vascularity. ${ }^{24}$ A role for TF in angiogenesis was also suggested by the finding that complete deletion of the TF gene in mice led to a defect in remodeling of the yolk sac vasculature and death at embryonic day 9.5. ${ }^{51}$ A subsequent study showed that development of the yolk sac vasculature required TFdependent thrombin generation and PAR1 activation on endothelial cells. ${ }^{52}$ However, it should be noted that the cellular processes involved in the development of the yolk sac vasculature and tumor angiogenesis may be very different. A recent study investigated the contribution of host-derived TF to tumor neovascularization in B16F1 melanoma and Lewis lung carcinoma cells. ${ }^{35}$ Growth of both of these tumors was not affected by reducing TF expression in the host, but B16F1 tumors in low-TF mice had smaller blood vessels compared with tumors in control mice.

\section{TF AND TUMOR GROWTH}

Much of the early work on TF expression in tumors was correlative and did not provide direct evidence that TF actively contributed to tumor growth. More recently, however, a study showed a direct role for TF in tumor growth. Strikingly, a selective reduction in TF expression in colorectal cancer cells using small interfering RNA dramatically reduced tumor growth in mice. ${ }^{33}$ Interestingly, the reduction of TF expression did not affect growth of the tumor cells in vitro, suggesting that TF-mediated enhancement of tumor growth requires a factor present in vivo that is not present when cells are grown in vitro. A likely candidate is FVIIa, which would form a TF:FVIIa complex on the surface of tumor cells in vivo leading to activation of PAR2dependent signaling. ${ }^{53}$ TF-dependent thrombin generation and activation of PAR1 on tumor cells may also increase tumor growth.

Inhibition of the TF:FVIIa complex either with a tissue factor pathway inhibitor or with a nematode anticoagulant protein called NAPc2 reduced growth of B16 melanomas in mice. ${ }^{54}$ Another study showed that a TF-blocking antibody reduced vascularization, VEGF expression, and growth of human carcinomas in immunodeficient mice. ${ }^{55}$ It was proposed that the inhibition of tumor growth may be mediated through a nonhemostatic, TF signaling-dependent mechanism because inhibition of the downstream coagulation serine protease FXa was ineffective. ${ }^{54}$ In support of this idea, selective inhibition of TF:FVIIa signaling using a monoclonal antibody 
called $10 \mathrm{H} 10$ reduced breast tumor growth in mice. ${ }^{56} 10 \mathrm{H} 10$ does not inhibit TF procoagulant activity. Finally, mice lacking PAR2, but not PAR1, exhibited reduced tumor growth in a model of spontaneous mammary tumors. ${ }^{57}$ Despite these studies, the precise mechanism by which the TF:FVIIa-PAR2 pathway enhances tumor growth remains to be fully elucidated. Activation of this pathway may increase tumor growth by directly enhancing tumor cell survival and/or by increasing the expression of proangiogenic proteins, such as VEGF and interleukin $8 .^{33,53,58}$

\section{TF AND ANTITUMOR STRATEGIES}

Two very different antitumor strategies have been developed that involve TF. The first targets a modified version of TF to the tumor vasculature where it induces local thrombosis. ${ }^{59}$ Thrombogens are chimeric proteins consisting of a targeting domain and a procoagulant domain. The targeting part of the chimera selectively recognizes the tumor vasculature and localizes the thrombogen to the tumor. An example of a targeting peptide is GNGRAHA, which targets aminopeptidase $\mathrm{N}$ and the integrin $\alpha \mathrm{v} \beta 3$ on the tumor vasculature. ${ }^{60}$ The procoagulant domain induces thrombosis within the tumor vasculature and the subsequent destruction of the tumor. A truncated form of $\mathrm{TF}$ ( $\mathrm{tTF}$ ) lacking the transmembrane domain is commonly used as the procoagulant domain. tTF has limited procoagulant activity in the circulation because it cannot localize FVIIa to a cell surface. However, it induces thrombosis when it becomes localized on the surface of the tumor vasculature via the targeting domain. A concern with this strategy is that adding the thrombogen to cancer patients with elevated levels of circulating TF may increase the risk of thrombosis.

The second strategy utilizes TF itself as a target for antitumor therapy. Some of the early work used an immunoconjugate molecule composed of a tumor-targeting domain conjugated to the Fc effector domain of human immunoglobulin G1. ${ }^{61-63}$ In this case, FVII was used to target TF-positive cells within the tumor, such as tumor cells themselves and possibly endothelial cells within the tumor vasculature. This strategy was designed to kill any cell that expressed TF. Direct injection of the immunoconjugate into the tumor was the preferred route of delivery to maximize tumor killing and minimize killing of TF-positive host cells that protect against bleeding. However, the main concern with this strategy remains bleeding, which could occur if the immunoconjugate escapes the tumor and enters the bloodstream.

Given that inhibition of the TF:FVIIa complex with either an anti-TF antibody or NAPc2 reduced tumor growth in mouse models, another potential approach is to use either an anti-TF antibody or a small molecule inhibitor of FVIIa to inhibit the TF:FVIIa complex in patients with cancer. This would inhibit both the procoagulant and signaling activities of the TF:FVIIa complex. In addition, as described above, selective inhibition of TF-dependent signaling may reduce tumor growth without inducing bleeding. Inhibition of PAR2 may result in a similar reduction in tumor growth. However, strategies that selectively block signaling of the TF:FVIIa-PAR2 pathway may not be as effective as those that block both signaling and procoagulant activities of TF because they will not impact thrombosis and metastasis. In summary, antitumor strategies involving TF hold promise and need to be evaluated further in the clinical setting.

\section{CONCLUSION}

There is compelling evidence that TF plays a role in many aspects of cancer biology including thrombosis, metastasis, tumor growth, and tumor angiogenesis. The use of circulating TF levels as biomarkers of thrombotic risk is currently being evaluated and could lead to the identification of a subset of patients that are at high risk for thrombosis. In the future, these patients could be treated with low molecular weight heparins or the new orally available anti-FXa and antithrombin anticoagulants. Finally, inhibition of the TF:VIIa complex holds promise as a novel approach to reduce both thrombosis and tumor growth.

\section{AUTHORS' DISCLOSURES OF POTENTIAL CONFLICTS OF INTEREST}

The author(s) indicated no potential conflicts of interest.

\section{AUTHOR CONTRIBUTIONS}

Manuscript writing: Raj S. Kasthuri, Mark B. Taubman, Nigel Mackman Final approval of manuscript: Nigel Mackman

\section{REFERENCES}

1. Mackman N: Role of tissue factor in hemostasis, thrombosis, and vascular development. Arterioscler Thromb Vasc Biol 24:1015-1022, 2004

2. Coughlin SR: Thrombin signaling and proteaseactivated receptors. Nature 407:258-264, 2000

3. Mackman $\mathrm{N}$ : Regulation of the tissue factor gene. FASEB J 9:883-889, 1995

4. Osterud B, Bjorklid E: Sources of tissue factor. Semin Thromb Hemost 32:11-23, 2006

5. Marmur JD, Rossikhina M, Guha A, et al: Tissue factor is rapidly induced in arterial smooth muscle after balloon injury. J Clin Invest 91:2253-2259, 1993

6. Solovey A, Gui L, Key NS, et al: Tissue factor expression by endothelial cells in sickle cell anemia. J Clin Invest 101:1899-1904, 1998

7. Mackman N, Tilley RE, Key NS: Role of the extrinsic pathway of blood coagulation in hemosta- sis and thrombosis. Arterioscler Thromb Vasc Biol 27:1687-1693, 2007

8. Morel O, Toti F, Hugel B, et al: Procoagulant microparticles: Disrupting the vascular homeostasis equation? Arterioscler Thromb Vasc Biol 26:25942604, 2006

9. Butenas S, Bouchard BA, Brummel-Ziedins $\mathrm{KE}$, et al: Tissue factor activity in whole blood. Blood 105:2764-2770, 2005

10. Giesen $P L$, Rauch $U$, Bohrmann B, et al: Blood-borne tissue factor: Another view of thrombosis. Proc Natl Acad Sci U S A 96:2311-2315, 1999

11. Mackman N: Alternatively spliced tissue factor one cut too many? Thromb Haemost 97:5-8, 2007

12. Nieuwland $R$, Berckmans RJ, RotteveelEijkman RC, et al: Cell-derived microparticles generated in patients during cardiopulmonary bypass are highly procoagulant. Circulation 96:3534-3541, 1997

13. Gregory SA, Morrissey JH, Edgington TS: Regulation of tissue factor gene expression in the monocyte procoagulant response to endotoxin. Mol Cell Biol 9:2752-2755, 1989

14. Morrissey JH, Drake TA, Schlag G: Procoagulant response of the endothelium and monocytes. New York, NY, Springer-Verlag, 1993

15. Pawlinski R, Pedersen B, Schabbauer G, et al: Role of tissue factor and protease-activated receptors in a mouse model of endotoxemia. Blood 103: 1342-1347, 2004

16. Parry GCN, Mackman N: Transcriptional regulation of tissue factor expression in human endothelial cells. Arterioscler Thromb Vasc Biol 15:612-621, 1995

17. Drake TA, Cheng J, Chang A, et al: Expression of tissue factor, thrombomodulin, and E-selectin in baboons with lethal Escherichia coli sepsis. Am J Pathol 142:1458-1470, 1993

18. Lupu C, Westmuckett AD, Peer G, et al: Tissue factor-dependent coagulation is preferentially up-regulated within arterial branching areas in a 
baboon model of Escherichia coli sepsis. Am J Pathol 167:1161-1172, 2005

19. Heit JA: Cancer and venous thromboembolism: Scope of the problem. Cancer Control 12:5-10, 2005 (suppl 1)

20. Sorensen HT, Mellemkjaer L, Olsen JH, et al: Prognosis of cancers associated with venous thromboembolism. N Engl J Med 343:1846-1850, 2000

21. Rickles FR, Hair GA, Zeff RA, et al: Tissue factor expression in human leukocytes and tumor cells. Thromb Haemost 74:391-395, 1995

22. Kakkar AK, Lemoine NR, Scully MF, et al: Tissue factor expression correlates with histological grade in human pancreatic cancer. $\mathrm{Br} \mathrm{J}$ Surg 82: 1101-1104, 1995

23. White $\mathrm{RH}$, Chew $\mathrm{H}$, Wun $\mathrm{T}$ : Targeting patients for anticoagulant prophylaxis trials in patients with cancer: Who is at highest risk? Thromb Res 120 S29-S40, 2007 (suppl 2)

24. Khorana $A A$, Ahrendt $S A$, Ryan $C K$, et al: Tissue factor expression, angiogenesis, and thrombosis in pancreatic cancer. Clin Cancer Res 13:28702875, 2007

25. Uno K, Homma S, Satoh T, et al: Tissue factor expression as a possible determinant of thromboembolism in ovarian cancer. Br J Cancer 96:290295, 2007

26. Rauch U, Antoniak S, Boots M, et al: Association of tissue-factor upregulation in squamous-cell carcinoma of the lung with increased tissue factor in circulating blood. Lancet Oncol 6:254, 2005

27. Del Conde I, Bharwani LD, Dietzen DJ, et al: Microvesicle-associated tissue factor and Trousseau's syndrome. J Thromb Haemost 5:70-74, 2007

28. Sato T, Tsujino I, Ikeda D, et al: Trousseau's syndrome associated with tissue factor produced by pulmonary adenocarcinoma. Thorax 61:1009-1010, 2006

29. Dvorak HF, Van DeWater L, Bitzer AM, et al: Procoagulant activity associated with plasma membrane vesicles shed by cultured tumor cells. Cancer Res 43:4434-4442, 1983

30. Ma L, Francia G, Viloria-Petit A, et al: In vitro procoagulant activity induced in endothelial cells by chemotherapy and antiangiogenic drug combinations: Modulation by lower-dose chemotherapy. Cancer Res 65:5365-5373, 2005

31. Swystun LL, Shin LY, Beaudin S, et al: Chemotherapeutic agents doxorubicin and epirubicin induce a procoagulant phenotype on endothelial cells and blood monocytes. J Thromb Haemost 7:619626, 2009

32. Lechner D, Kollars M, Gleiss A, et al: Chemotherapy-induced thrombin generation via procoagulant endothelial microparticles is independent of tissue factor activity. J Thromb Haemost 5:24452452, 2007

33. $\mathrm{Yu}$ JL, May L, Lhotak $\mathrm{V}$, et al: Oncogenic events regulate tissue factor expression in colorectal cancer cells: Implications for tumor progression and angiogenesis. Blood 105:1734-1741, 2005

34. Davila M, Amirkhosravi A, Coll E, et al: Tissue factor-bearing microparticles derived from tumor cells: Impact on coagulation activation. J Thromb Haemost 6:1517-1524, 2008

35. Yu J, May L, Milsom C, et al: Contribution of host-derived tissue factor to tumor neovascularization. Arterioscler Thromb Vasc Biol 28:1975-1981, 2008

36. Tesselaar ME, Romijn FP, Van Der Linden IK, et al: Microparticle-associated tissue factor activity: A link between cancer and thrombosis? J Thromb Haemost 5:520-527, 2007

37. Hron G, Kollars M, Weber $H$, et al: Tissue factor-positive microparticles: Cellular origin and association with coagulation activation in patients with colorectal cancer. Thromb Haemost 97:119-123, 2007

38. Khorana AA, Francis CW, Menzies KE, et al: Plasma tissue factor may be predictive of venous thromboembolism in pancreatic cancer. J Thromb Haemost 6:1983-1985, 2008

39. Amirkhosravi A, Meyer T, Chang JY, et al: Tissue factor pathway inhibitor reduces experimental lung metastasis of B16 melanoma. Thromb Haemost 87:930-936, 2002

40. Mueller BM, Reisfeld RA, Edgington TS, et al: Expression of tissue factor by melanoma cells promotes efficient hematogenous metastasis. Proc Natl Acad Sci U S A 89:11832-11836, 1992

41. Mueller BM, Ruf W: Requirement for binding of catalytically active factor VIla in tissue factordependent experimental metastasis. J Clin Invest 101:1372-1378, 1998

42. Camerer E, Qazi AA, Duong DN, et al: Platelets, protease-activated receptors, and fibrinogen in hematogenous metastasis. Blood 104:397-401, 2004

43. Palumbo JS, Kombrinck KW, Drew AF, et al: Fibrinogen is an important determinant of the metastatic potential of circulating tumor cells. Blood 96:3302-3309, 2000

44. Seto $S$, Onodera H, Kaido $T$, et al: Tissue factor expression in human colorectal carcinoma: Correlation with hepatic metastasis and impact on prognosis. Cancer 88:295-301, 2000

45. Palumbo JS, Talmage KE, Massari JV, et al: Tumor cell-associated tissue factor and circulating hemostatic factors cooperate to increase metastatic potential through natural killer cell-dependent and-independent mechanisms. Blood 110:133-141, 2007

46. Palumbo JS, Talmage KE, Massari JV, et al: Platelets and fibrin(ogen) increase metastatic potential by impeding natural killer cell-mediated elimination of tumor cells. Blood 105:178-185, 2005

47. Contrino J, Hair G, Kreutzer DL, et al: In situ detection of tissue factor in vascular endothelial cells: Correlation with the malignant phenotype of human breast disease. Nat Med 2:209-215, 1996

48. Luther T, Flossel C, Mackman N, et al: Tissue factor expression during human and mouse development. Am J Pathol 149:101-113, 1996

49. Shoji M, Hancock WW, Abe K, et al: Activation of coagulation and angiogenesis in cancer: Immunohistochemical localization in situ of clotting proteins and vascular endothelial growth factor in human cancer. Am J Pathol 152:399-411, 1998

50. Zhang $Y$, Deng $Y$, Luther $T$, et al: Tissue factor controls the balance of angiogenic and antiangiogenic properties of tumor cells in mice. J Clin Invest 94:1320-1327, 1994

51. Carmeliet $P$, Mackman $N$, Moons $L$, et al: Role of tissue factor in embryonic blood vessel development. Nature 383:73-75, 1996

52. Griffin CT, Srinivasan $Y$, Zheng $Y W$, et al: A role for thrombin receptor signaling in endothelial cells during embryonic development. Science 293: 1666-1670, 2001

53. Ruf $W$ : Tissue factor and PAR signaling in tumor progression. Thromb Res 120:S7-S12, 2007 (suppl 2)

54. Hembrough TA, Swartz GM, Papathanassiu A, et al: Tissue factor/factor VIla inhibitors block angiogenesis and tumor growth through a nonhemostatic mechanism. Cancer Res 63:2997-3000, 2003

55. Milsom CC, Yu JL, Mackman N, et al: Tissue factor regulation by epidermal growth factor receptor and epithelial-to-mesenchymal transitions: Effect on tumor initiation and angiogenesis. Cancer Res 68:10068-10076, 2008

56. Versteeg HH, Schaffner F, Kerver M, et al: Inhibition of tissue factor signaling suppresses tumor growth. Blood 111:190-199, 2008

57. Versteeg HH, Schaffner $F$, Kerver $M$, et al: Protease-activated receptor (PAR) 2, but not PAR1, signaling promotes the development of mammary adenocarcinoma in polyoma middle $\mathrm{T}$ mice. Cancer Res 68:7219-7227, 2008

58. Hjortoe GM, Petersen LC, Albrektsen T, et al: Tissue factor-factor VIla-specific up-regulation of IL-8 expression in MDA-MB-231 cells is mediated by PAR-2 and results in increased cell migration. Blood 103:3029-3037, 2004

59. Huang X, Molema G, King S, et al: Tumor infarction in mice by antibody-directed targeting of tissue factor to tumor vasculature. Science 275:547550, 1997

60. Bieker R, Kessler T, Schwoppe C, et al: Infarction of tumor vessels by NGR-peptide directed targeting of tissue factor: Experimental results and first-in-man experience. Blood 113:5019-5027, 2009

61. Hu Z, Garen A: Intratumoral injection of adenoviral vectors encoding tumor-targeted immunoconjugates for cancer immunotherapy. Proc Natl Acad Sci U S A 97:9221-9225, 2000

62. Hu Z, Garen A: Targeting tissue factor on tumor vascular endothelial cells and tumor cells for immunotherapy in mouse models of prostatic cancer. Proc Natl Acad Sci U S A 98:12180-12185, 2001

63. Hu Z, Sun Y, Garen A: Targeting tumor vasculature endothelial cells and tumor cells for immunotherapy of human melanoma in a mouse xenograft model. Proc Natl Acad Sci U S A 96:8161-8166, 1999 